\title{
Micromodel Study on Pore-Scale Mechanisms Associated with Permeability Impairment in Porous media
}

Safna Nishad and Riyadh I. Al-Raoush

Department of Civil \& Architectural Engineering, Qatar University, P.O Box 2713, Doha, Qatar

\section{BACKGROUND AND MOTIVATION}

$\checkmark$ Methane Hydrate bearing sediments are potential energy resource reserving 500-1000Gt of carbon world wide.

$\checkmark$ Dissociation of hydrate into gas and water is the preliminary process in gas production.

$\checkmark$ Mobilization and migration of fines during gas production reduce the permeability of the formation.

$\checkmark$ Laboratory column studies reveal the reduction in permeability during single and two-phase flow.

$\checkmark$ Clogging of pore throats by fines significantly decrease permeability.

The fines mobilized by the gas-water interface might impact the permeability.

$\checkmark$ Pore-scale visualization studies need to be conducted to understand the mechanisms responsible for permeability reduction during two-phase flow.

\section{OBJECTIVES}

The objective of this study was to investigate the pore-scale mechanisms associated with fines mobilization on permeability reduction during two-phase flow using a micromodel.

\section{EXPERIMENTAL STUDY}

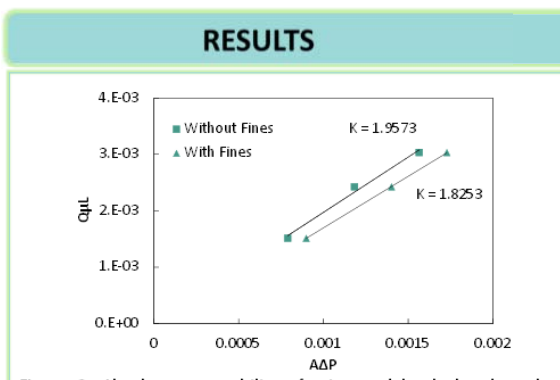

Figure 2: Absolute permeability of micromodel calculated as the slope of volumetric discharge and pressure drop before and after fine injection

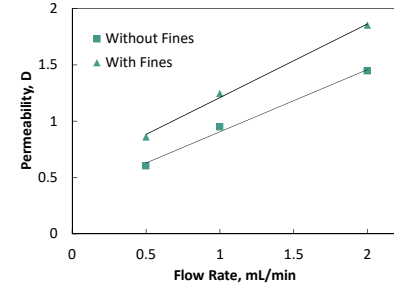

Figure 3: Variation of effective permeability of $\mathrm{CO}_{2}$ with the flow rate before and after fine injection

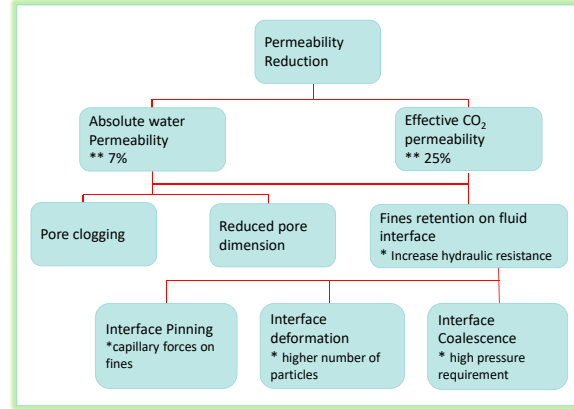

$\checkmark$ Breakthrough pressure of $\mathrm{CO} 2$ increased $39 \%$ with the presence of fines

$\checkmark$ Based on the pore-scale observations, the reduction in permeability is affected by the following factors:

$\checkmark$ Percentage of particles in the porous media: increased retention on gas-water interfaces

$\checkmark$ Type of particles (i.e., hydrophilic or hydrophobic): the strength of capillary forces holding the particles on fluid interface increases with decreasing hydrophobicity

Solution chemistry (i.e., ionic strength, $\mathrm{pH}$ etc.): the adhesion forces on solid surfaces are impacted by surface forces
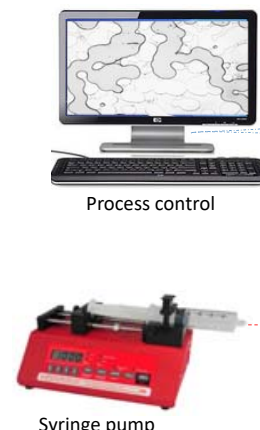

Figure 1: Experimental Set-up
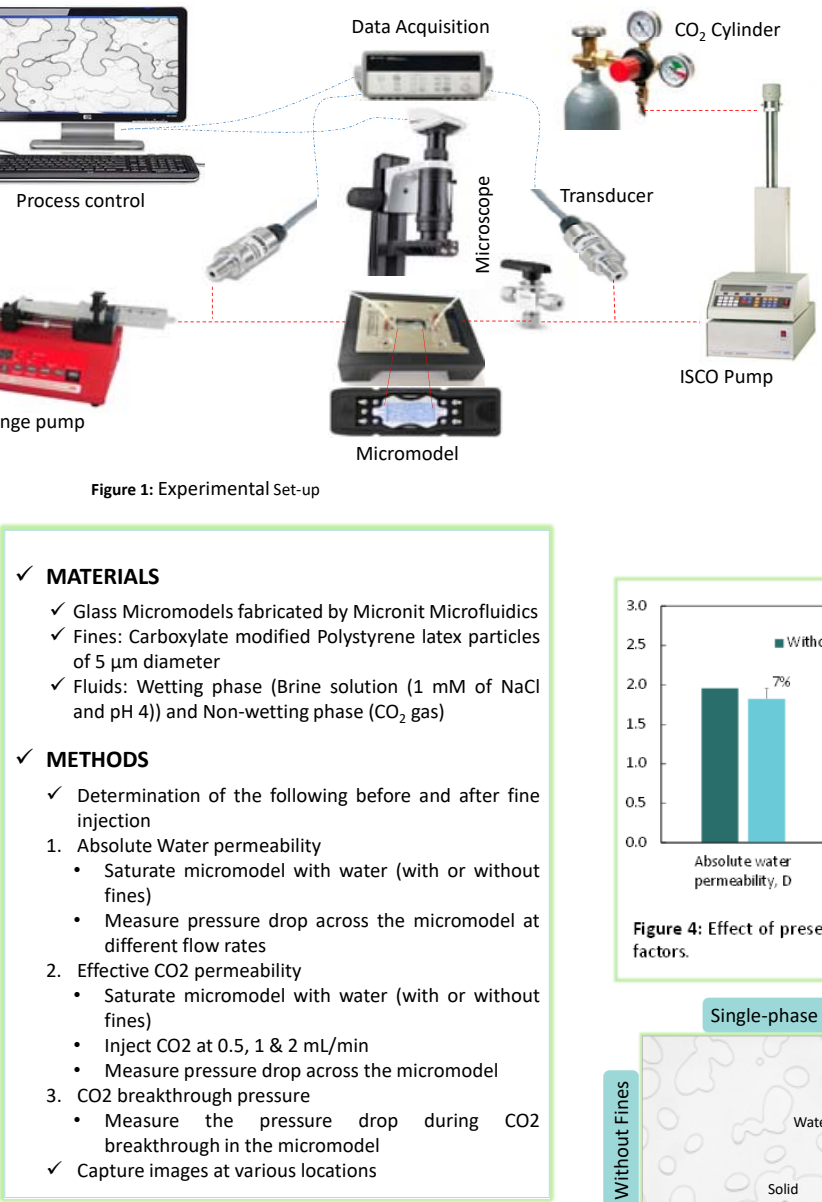

\section{Viscosity of water (cP), $\mu_{w}$}

Viscosity of $\mathrm{CO}_{2}(\mathrm{cP}), \mathrm{\mu}_{\mathrm{CO} 2}$

Area $\left(\mathrm{cm}^{2}\right)$, A

Length $(\mathrm{cm})$, L

0.91

0.01473

0.002

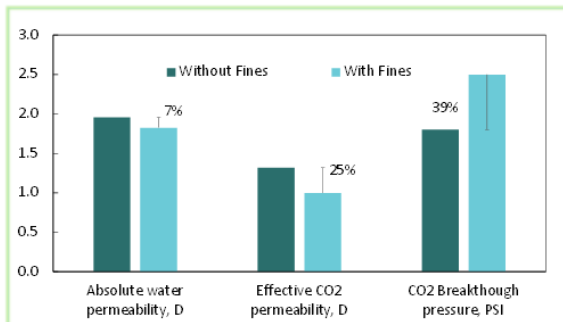

Figure 4: Effect of presence of fines in the micromodel on different factors.

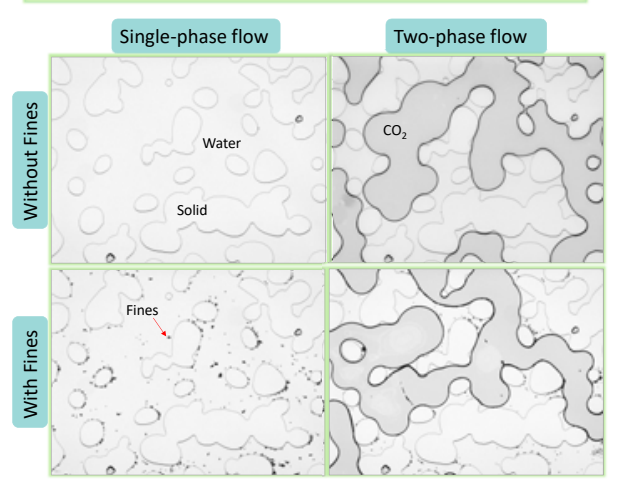

Figure 5: Micromodel images at different permeability measurement conditions

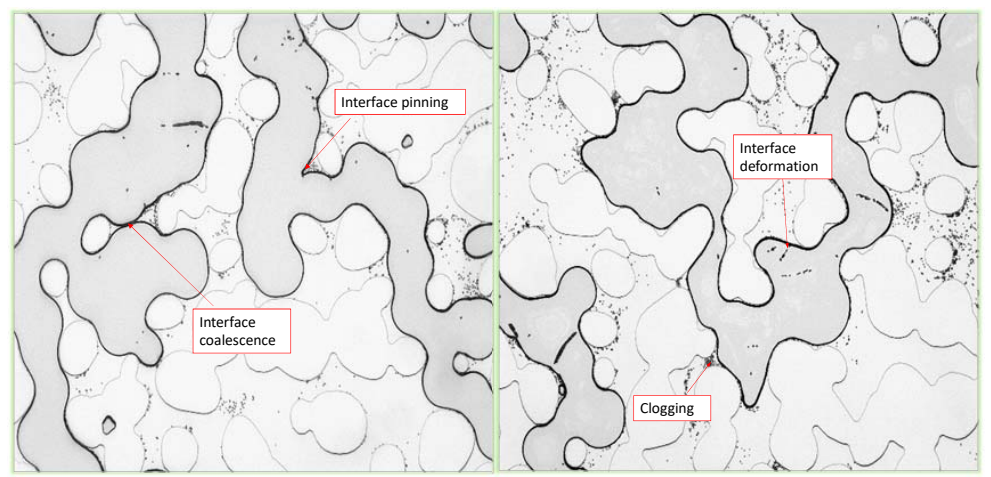

Figure 6: Different mechanisms affecting permeability of the porous media; interface pinning, coalescence and deformation in addition to pore clogging

\section{CONCLUSIONS}

The important findings from the experimental investigation are summarized below:

- There is a decrease in permeability of the porous media due to the presence of fine particles.

The reduction in permeability during single-phase flow is due to the pore clogging or decreased pore dimensions by the presence of fines.

- Comparatively higher percentage reduction in effective permeability during two-phase flow with $\mathrm{CO}_{2}$.

> The resistance to invade the pores by the gas-water interface increased by the presence of fines in the pore space.

- Fine particles attached on solid surface are mobilized with the moving gas-water interface and retained on the interface.

> The capillary retention of fines on gas-water interface pin the interface on grain surface causing higher hydraulic resistance to flow.

The coalescence of two moving interfaces require higher capillary pressure due to the presence of fines on the interface.

> The different pore scale mechanisms (i.e., interface pinning, deformation and coalescence) are responsible for increase reduction in permeability during two-phase flow.

- The permeability can be affected by the percentage and type of fines and fluid chemistry in the porous medium.

\section{ACKNOWLEDGMENT}

